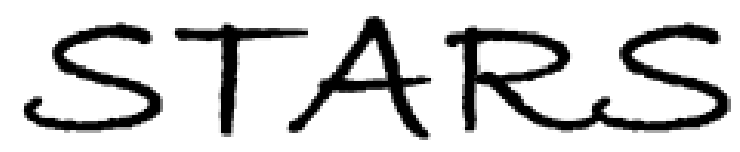

University of Central Florida

STARS

$1-1-1997$

\title{
Spin-ensemble density-functional theory for inhomogeneous quantum Hall systems
}

\author{
M. I. Lubin \\ University of Central Florida \\ O. Heinonen \\ University of Central Florida \\ M. D. Johnson \\ University of Central Florida
}

Find similar works at: https://stars.library.ucf.edu/facultybib1990

University of Central Florida Libraries http://library.ucf.edu

This Article is brought to you for free and open access by the Faculty Bibliography at STARS. It has been accepted for inclusion in Faculty Bibliography 1990s by an authorized administrator of STARS. For more information, please contact STARS@ucf.edu.

\section{Recommended Citation}

Lubin, M. I.; Heinonen, O.; and Johnson, M. D., "Spin-ensemble density-functional theory for inhomogeneous quantum Hall systems" (1997). Faculty Bibliography 1990s. 2001.

https://stars.library.ucf.edu/facultybib1990/2001

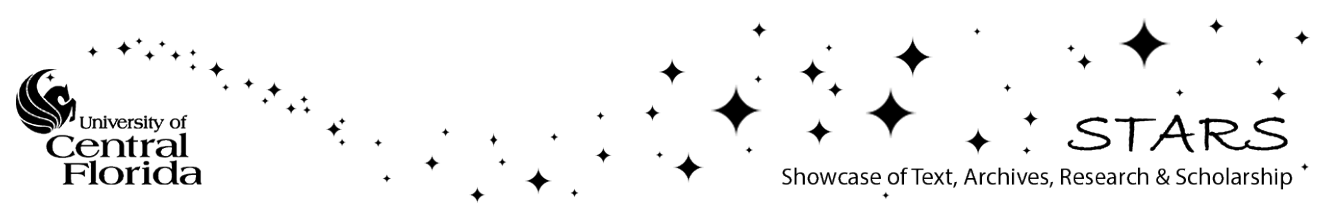




\title{
Spin-ensemble density-functional theory for inhomogeneous quantum Hall systems
}

\author{
M. I. Lubin, O. Heinonen, and M. D. Johnson \\ Department of Physics, University of Central Florida, Orlando, Florida 32816-2385
}

(Received 10 January 1997; revised manuscript received 14 July 1997)

\begin{abstract}
We have developed an ensemble density-functional theory that includes spin degrees of freedom for nonuniform quantum Hall systems. We have applied this theory using a local-spin-density approximation to study the edge reconstruction of parabolically confined quantum dots. For a Zeeman splitting below a certain critical value, the edge of a completely polarized maximum density droplet reconstructs into a spin-unpolarized structure. For larger Zeeman splittings, the edge remains polarized and develops an exchange hole.
\end{abstract}

[S0163-1829(97)07240-8]

\section{INTRODUCTION}

In this paper we develop a spin-ensemble densityfunctional approach for the study of inhomogeneous quantum Hall systems. This permits the study of properties, such as edge structures, of systems with many electrons. (Some results for spin-polarized systems were reported earlier. ${ }^{1-3}$ ) We show here that fractional Hall systems cannot be described using conventional density-functional approaches, but that the generalization known as ensemble densityfunctional theory is a very suitable tool. For spin-polarized systems our implementation of ensemble density-functional theory gives results in excellent agreement with other approaches in limits where comparisons can be made (e.g., the semiclassical limit, and the limit of small system size). A generalization to include the spin degree of freedom lets us study the interplay of density and spin during edge reconstructions in realistically large systems.

The quantum Hall effects (QHE's) occur ${ }^{4}$ in a twodimensional electron gas in a magnetic field $\mathbf{B}=B \hat{z}$ perpendicular to the electron system. ${ }^{5}$ A quantum treatment of the motion of an infinite, homogeneous system shows that the kinetic energy takes discrete values $(n+1 / 2) \hbar \omega_{c}$, where $n$ is the Landau-level index $(n=0,1,2, \ldots)$ and $\omega_{c}=e B / m^{*} c$ is the cyclotron frequency $\left(m^{*}\right.$ is the effective mass of an electron). Each Landau level contains $n_{B}=B / \Phi_{0}$ states per unit area, or one state for each magnetic-flux quantum $\Phi_{0}=h c / e$, giving rise to a macroscopic Landau-level degeneracy. The ratio of the electron areal density $n(\mathbf{r})$ to $n_{B}$ defines the filling factor $\nu(\mathbf{r})=n(\mathbf{r}) / n_{B}$. The filling factor also can be expressed as $\nu=2 \pi l_{B}^{2} n$, where $l_{B}=\sqrt{\hbar c / e B}$ is the magnetic length.

For an explanation of experimental studies it is highly desirable to have a computational approach that accurately treats systems with of the order of $1-10^{3}$ electrons, and that can include effects such as accurate confinements, spin degrees of freedom, and finite layer thickness. Exact numerical diagonalizations are limited to very small systems $(N \lesssim 10){ }^{6,7}$ Semiclassical methods ${ }^{8,9}$ do not accurately treat electron-electron interactions, and effective field theories ${ }^{10}$ cannot give accurate quantitative information about many system properties. A method that can deal with a larger number of electrons is the composite fermion theory in the Har- tree approximation. ${ }^{11}$ However, in this approach, the singular Chern-Simons gauge field is replaced by its smooth spatial average, and the composite fermion mass has to be put into the calculations by hand. Furthermore, interpretation of the results is sometimes difficult and ambiguous. On the other hand, density-functional theory (DFT) is known as a general quantitative method to include exchange-correlation effects in inhomogeneous systems without any fitting parameters. In this paper we show that ensemble DFT can be used for quantum Hall systems with highly accurate results.

DFT was originally formulated by Hohenberg and Kohn as a practical method for a description of the ground-state properties of many-body systems. ${ }^{12}$ The foundation of DFT is the Hohenberg-Kohn theorem, which states that the ground-state density uniquely determines the Hamiltonian of a system (to within a constant). Furthermore, a variational principle states that the ground-state density minimizes the energy of the system. We will use the constrained search formulation of Levy ${ }^{13}$ for the Hohenberg-Kohn theorem and its associated variational principle. In this elegant approach the ground-state energy $E$ can be written as a functional of the density,

$$
E[n]=F[n]+\int d \mathbf{r} n(\mathbf{r}) V_{\mathrm{ext}}(\mathbf{r})
$$

Here

$$
F[n]=\inf _{\Psi \rightarrow n}\left\langle\Psi\left|\hat{T}+\hat{V}_{\mathrm{ee}}\right| \Psi\right\rangle,
$$

with $\hat{T}, \hat{V}_{\text {ee }}$, and $\hat{V}_{\text {ext }}$ kinetic energy, electron-electron interactions, and external potential, respectively. ${ }^{13}$ The infimum is taken over all many-body states $\Psi$ that yield a fixed density $n(\mathbf{r}) . F[n]$ so defined is then a universal functional of the density $n(\mathbf{r})$. For a given external potential $V_{\text {ext }}$, the true ground-state density is the function $n(\mathbf{r})$, which minimizes $E[n]$ in Eq. (1).

Practical computations using DFT are typically done by introducing an auxiliary noninteracting system with a ground-state density $n_{s}(\mathbf{r})$, and by asserting that there exists an effective potential $V_{s}(\mathbf{r})$ for this system such that $n_{s}(\mathbf{r})=n(\mathbf{r})$, with $n(\mathbf{r})$ the ground-state density of the real, interacting system. The density is then obtained from a Slater determinant of the Kohn-Sham (KS) orbitals $\psi_{\alpha}(\mathbf{r})$, 
$n_{s}(\mathbf{r})=\sum_{\alpha=1}^{N}\left|\psi_{\alpha}(\mathbf{r})\right|^{2}$, which are obtained by selfconsistently solving the KS equations ${ }^{14}$

$$
h_{\text {eff }} \psi_{\alpha}(\mathbf{r})=\left[T+V_{s}(\mathbf{r})\right] \psi_{\alpha}(\mathbf{r})=\varepsilon_{\alpha} \psi_{\alpha}(\mathbf{r}) .
$$

The self-consistency is achieved in practice by iteratively obtaining the eigenstates and occupying the $N$ eigenstates with the lowest eigenvalues $\varepsilon_{\alpha}$. The effective potential $V_{s}(\mathbf{r})$ can be derived from the Hohenberg-Kohn theorem and its associated variational principle. The result is

$$
V_{s}(\mathbf{r})=V_{\text {ext }}(\mathbf{r})+V_{\mathrm{H}}(\mathbf{r})+V_{\mathrm{xc}}(\mathbf{r}) .
$$

Here, $V_{\mathrm{H}}(\mathbf{r})$ is the classical Hartree potential, and $V_{\mathrm{xc}}(\mathbf{r})$ is the exchange-correlation potential. In practical calculations, the local-density approximation (LDA) is often used. ${ }^{14}$ In this approximation, one writes

$$
E_{\mathrm{xc}}^{\mathrm{LDA}}=\int d \mathbf{r} n(\mathbf{r}) \epsilon_{\mathrm{xc}}(n(\mathbf{r})),
$$

where $\epsilon_{\mathrm{xc}}(n)$ is the exchange-correlation energy per particle of an infinite, homogeneous system of density $n$. The exchange-correlation potential is then obtained as

$$
V_{\mathrm{xc}}^{\mathrm{LDA}}(\mathbf{r})=\left.\frac{d\left[n \epsilon_{\mathrm{xc}}(n)\right]}{d n}\right|_{n=n(r)} .
$$

Systems for which the ground-state density can be represented by a single ground-state wave function (whether the ground state is degenerate or not) are called pure-state $v$ representable. There are systems that are known not to be pure-state $v$ representable, as shown first by Levy ${ }^{15}$ and Lieb. ${ }^{16}$ However, there exists a generalization of the Hohenberg-Kohn theorem that provides a one-to-one correspondence between a ground-state density $n(\mathbf{r})$ and the Hamiltonian even for systems that are not pure-state $v$ representable, but whose density can be obtained from an ensemble of degenerate ground states. The functional $F[n]$ in Eq. (2) is extended to include ensembles over degenerate ground states $\left|\Psi_{i}\right\rangle, \hat{D}=\sum_{i=1}^{q} d_{i}\left|\Psi_{i}\right\rangle\left\langle\Psi_{i}\right|$, with $d_{i}=d_{i}^{*} \geqslant 0$, $\sum_{i=1}^{q} d_{i}=1$. This then yields the generalization

$$
F_{E}[n]=\inf _{\hat{D} \rightarrow n} \operatorname{Tr}\left\{\hat{D}\left(\hat{T}+\hat{V}_{\mathrm{ee}}\right)\right\},
$$

with the infimum taken over all $\hat{D}$ yielding a fixed density $n(\mathbf{r})$. There is then a generalized variational principle that states that $F_{E}[n]$ is minimized by the ground-state density, which can now be represented by an ensemble of wave functions, even if it cannot be represented by a single ground state. This generalization is called ensemble densityfunctional theory. The key question for a practical calculation is whether the conventional KS scheme can be applied. In fact, this requires a stronger criterion than noninteracting pure-state $v$ representability, which guarantees only that some superposition of noninteracting ground states yield the desired density. But to use the conventional KS scheme it is necessary that the density be noninteracting $v$ representable by a single Slater determinant. As we shall see below in Sec. II, fractional QHE systems are systems in which there is a huge degeneracy in the KS orbitals, and the density cannot in general be obtained from a single Slater determinant of KS orbitals, so an ensemble DFT has to be used.

In Sec. III we present a test of the accuracy of our ensemble DFT scheme by applying it to spin-polarized quantum Hall dots and comparing with numerical diagonalizations. In GaAs samples, where most of QHE experiments have been done, the spin degree of freedom is important, and may lead to inhomogeneous spin densities. The small magnitude of the Landé factor $g^{*}$ (about 0.44 in GaAs) makes the existence of partly polarized states energetically possible ${ }^{17,18}$ even at $\nu<1$. In Sec. IV we use our scheme to describe the spin-textured edge reconstruction of $\nu=1$ quantum Hall dots. Finally, conclusions are given in Sec. V.

\section{II. $V$ REPRESENTABILITY AND THE QUANTUM HALL EFFECT}

In practical ensemble DFT calculations one introduces as in the KS scheme an auxiliary noninteracting system that provides the basis for the density matrix and has a groundstate density identical to the interacting system at hand. The variational principle again yields ${ }^{19}$ the KS equations, Eq. (3). However, the density for $N$ electrons is now given by

$$
n(\mathbf{r})=\sum_{\alpha} f_{\alpha}\left|\psi_{\alpha}(\mathbf{r})\right|^{2}, \quad \sum_{\alpha} f_{\alpha}=N,
$$

with the occupation numbers $f_{\alpha}$ in the interval $0 \leqslant f_{\alpha} \leqslant 1$. One obtains fractional occupancies $f_{\alpha}$ only when the corresponding KS eigenvalues $\varepsilon_{\alpha}$ are degenerate and equal to the Fermi energy $\varepsilon_{F}$. (If $\varepsilon_{\alpha}<\varepsilon_{F}$, then $f_{\alpha}=1$.)

Let us show briefly why applying DFT to the fractional quantum Hall effect (FQHE) inevitably requires ensemble DFT. Consider a circularly symmetric $N$-particle FQHE system with a uniform density corresponding to $\nu=1 / 3$ out to some radius $r_{0} \approx \sqrt{6 N} l_{B}$. (At the edge the density falls to zero within a distance of order $l_{B}$. We will ignore details near the edge, which are irrelevant for our discussion.) That such systems exist is well demonstrated by the excellent agreement between the Laughlin wave function and experiments, and by many numerical calculations. ${ }^{20,21}$ We wish to model this interacting system with an auxiliary noninteracting system using DFT. To do so we must establish that there exists a unique effective single-particle potential $V_{s}$ that gives a (possibly degenerate) ground state $\Psi$ with the desired density. We can readily establish that the desired $V_{s}$ is simply a constant by using the generalization of the HohenbergKohn theorem to degenerate ground states, as follows. We first choose $V_{s}(\mathbf{r})=$ const, and we will show that this choice of $V_{s}$ will yield a ground state with the correct density. Due to the circular symmetry the KS orbitals are the usual symmetric gauge eigenstates $\psi_{n m}(\mathbf{r})$, with $n$ the Landau-level index and $m$ angular momentum. For a given $n$ these orbitals are degenerate in $m$. Consequently every $N$-particle Slater determinant made up of any $N$ lowest-Landau-level $(n=0)$ orbitals is a ground state- the noninteracting ground state is hugely degenerate. We now explicitly demonstrate that we can construct a ground state with the desired density. For example, we may choose the particular superposition of determinants that yields the Laughlin wave function. Or, more simply, choose $\Psi=(1 / \sqrt{3})\left(\Psi_{1}+\Psi_{2}+\Psi_{3}\right)$, where 
$\Psi_{1}, \Psi_{2}, \Psi_{3}$ are the Slater determinants made up of lowest-Landau-level orbitals with, respectively, $\{m\}$ $=\{0,3,6, \ldots\},\{1,4,7, \ldots\},\{2,5,8, \ldots\}$. Both choices yield $\mathrm{N}$-particle wave functions with the desired density corresponding to $\nu=1 / 3$ in the bulk.

Thus there exists a potential $V_{s}(\mathbf{r})=$ const for which a noninteracting ground state yields the correct density. The generalized Hohenberg-Kohn theorem then ensures that this potential is unique (to within a constant). Since we must use this $V_{s}$, we must construct the density in the noninteracting system from the orbitals $\psi_{n m}$. But to get the right density requires fractional occupancy of these orbitals. (For example, consider the origin: all lowest-Landau-level orbitals with $m \neq 0$ vanish at the origin, and $\psi_{00}$ has a local filling factor of unity at $r=0$. Hence to get a filling factor $\nu=1 / 3$ at $r=0$ requires that $\psi_{00}$ have occupancy $1 / 3$.) This example leads to the key result of this section: it is impossible to construct the FQHE ground-state density from a single Slater determinant of KS orbitals. Hence the conventional KS approach cannot be used, and we must turn to ensemble density-functional theory.

It is evident from this example that the FQHE may in general be noninteracting pure-state $v$ representable, i.e., the ground-state density can be extracted from a single noninteracting ground state consisting of a sum over degenerate Slater determinants. However, this is not a very useful statement for practical purposes, where the important consideration is whether or not the ground-state density can be constructed from a single ground-state Slater determinant of KS orbitals. Whenever the KS orbitals have degeneracies that lead to degenerate ground states of the noninteracting system, the conventional KS scheme cannot be used and one needs to try ensemble density-functional theory.

In inhomogeneous QHE systems, which may include regions with locally fractional and integer fillings, not all KS orbitals are degenerate, but there does exist a set of degenerate orbitals at the Fermi energy. By a simple extension of the above argument, ensemble DFT must be used for these systems, too. For inhomogeneous systems one finds $M$ orbitals with $\varepsilon_{\alpha}<\varepsilon_{F}$ and $D$ degenerate orbitals with $\varepsilon_{\alpha}=\varepsilon_{F}$. One constructs determinantal wavefunctions $\Psi_{i}$ in which all $M$ low-energy orbitals are occupied; the $\Psi_{i}$ differ by which $N-M$ of the $D$ degenerate orbitals are occupied. With $\theta_{i \alpha}$ denoting the occupancy of orbital $\alpha$ in the determinant $\Psi_{i}$, the total density of the ensemble can then be written

$$
n(\mathbf{r})=\sum_{\alpha} \sum_{i=1}^{q} d_{i} \theta_{i \alpha}\left|\psi_{\alpha}(\mathbf{r})\right|^{2}
$$

where $d_{i}$ is the weight of $\Psi_{i}$ in the ensemble. Comparing the result with Eq. (8), one can see how the fractional occupational numbers $f_{\alpha}$ of the degenerate KS orbitals follow from the weights $d_{i}$ in the expansion of density matrix:

$$
f_{\alpha}=\sum_{i=1}^{q} d_{i} \theta_{i \alpha}
$$

If the ground-state density can be written in this form, then one can appeal to the ensemble generalization of the Hohenberg-Kohn theorem and its generalized variational principle, as explained in Sec. I. However, a procedure to compute the fractional occupancies $f_{\alpha}$ has not existed, ${ }^{22}$ and one major advance in our work is that we have found a simple way to generate the occupancies, at least for the FQHE. (The procedure is explained in our earlier work. ${ }^{1,2}$ ) Applying ensemble DFT to the FQHE, we have found that fractionally occupied KS orbitals are indeed degenerate at the Fermi energy, consistent with our demonstration above that the ground-state density of an FQHE cannot in general be obtained from a single ground-state Slater determinant of KS orbitals.

\section{SPIN-POLARIZED QUANTUM DOT IN A FQHE REGIME}

Quantum dots can contain from one to several thousand electrons, typically with very inhomogeneous density distributions. Moreover, quantum dots are believed to have highly correlated ground states in strong magnetic fields. ${ }^{23}$ Hence these are ideal systems to examine the usefulness of our ensemble DFT approach, including the important question of how well the strong correlation effects are included compared, say, with exact diagonalization studies.${ }^{6,7}$ In this section we will use a small-system spin-polarized quantum dot as a test case to study our ensemble DFT approach and to compare our results with those obtained by numerical diagonalizations. ${ }^{6}$ (We refer to Refs. 1-3 for an explanation of our ensemble DFT-LDA scheme and results for larger spin-polarized quantum Hall systems.) One of the things considered is the reconstruction of the so-called maximum density droplet (MDD), which is the most compact droplet (minimum angular momentum) droplet that can be formed in the lowest Landau level.

We have calculated the expectation value of the total angular momentum $\langle M\rangle=\Sigma_{m n} m f_{m n}$ as a function of the magnetic-field strength $B$ for $N=6$ spin-polarized electrons in the lowest Landau level and a parabolic confining potential $V_{\text {ext }}=\frac{1}{2} m * \Omega^{2} r^{2}$, with $\hbar \Omega=2.0 \mathrm{meV}$. The results are shown in Fig. 1 for two different versions of the exchangecorrelation energy $\epsilon_{\mathrm{xc}}$. The diamonds $(\diamond)$ were generated using the Levesque-Weiss-MacDonald exchange-correlation energy, ${ }^{24}$ while the pluses $(+)$ were generated using an exchange-correlation energy due to Fano and Ortolani. ${ }^{25}$ This latter uses $\epsilon_{\mathrm{xc}}$ at $\nu \leqslant 1 / 2$ and the particle-hole symmetry of the lowest Landau level to give a good interpolation formula on the entire interval $0 \leqslant \nu \leqslant 1$. Both exchangecorrelation energies give clear plateaus or plateaulike structures in angular momentum vs magnetic field. However, the Levesque-Weiss-MacDonald is a rather poor approximation near $\nu=1$ (a region for which it was not constructed), and furthermore, overestimates the magnitude of the exchangecorrelation potential at about $\nu=1 / 2$. As a consequence, the initial maximum density droplet instability is smeared out and starts at a too low a value of magnetic field, and the formation of a 1/3 droplet (as is evidenced by studies of the density profile) occurs at a too high value of magnetic field. Also, the values of the angular momentum at the plateaulike regions tend to be too low. For example, the formation of the $1 / 3$ droplet occurs at $M \approx 40$, while the exact value is $M=45$. In contrast, the results obtained using Fano-Ortolani exchange-correlation energy tend to be very accurate. For example, the maximum density droplet instability occurs at 


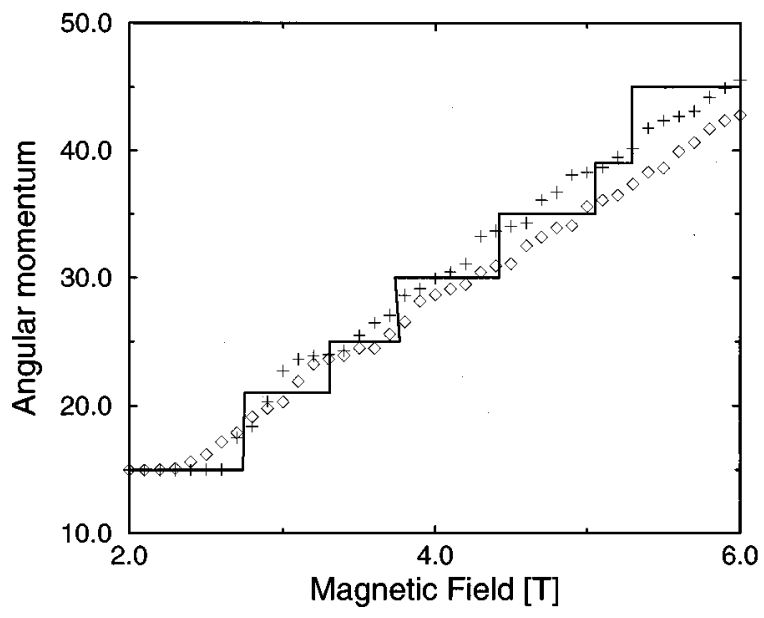

FIG. 1. Expectation value of the total angular momentum $\langle M\rangle=\Sigma_{m n} m f_{m n}$ as a function of the magnetic-field strength $B$ indicated by solid line for a spin-polarized six-electron droplet in a parabolic confinement using the Levesque-Weiss-MacDonald (Ref. 24) $(\diamond)$ and Fano-Ortolani (Ref. 25) (+) exchange-correlation energies. The solid shows the exact diagonalization studies result from Ref. 6.

$B \approx 2.8 \mathrm{~T}$ in our calculations, compared to $B=2.75 \mathrm{~T}$ in the numerical diagonalizations, and the 1/3-droplet formation occurs at $B \approx 5.3 \mathrm{~T}$ in our calculations, compared to $B=5.29 \mathrm{~T}$ in the numerical diagonalizations (see Fig. 2). In addition, the plateaulike regions are more developed and flatter in angular momentum. Still, though, the ensemble DFT tends to underestimate the angular momentum at the plateaus. We want to emphasize here that we have not used any adjusting parameters in our calculations. Furthermore, the ensemble DFT is not constructed only to give integer angular momenta. Finally, only the energy gaps at $\nu=1 / 3$ and $\nu=2 / 5$, along with their particle-hole conjugates at $\nu=2 / 3$ and $\nu=3 / 5$ were included, while the numerical diagonaliza-

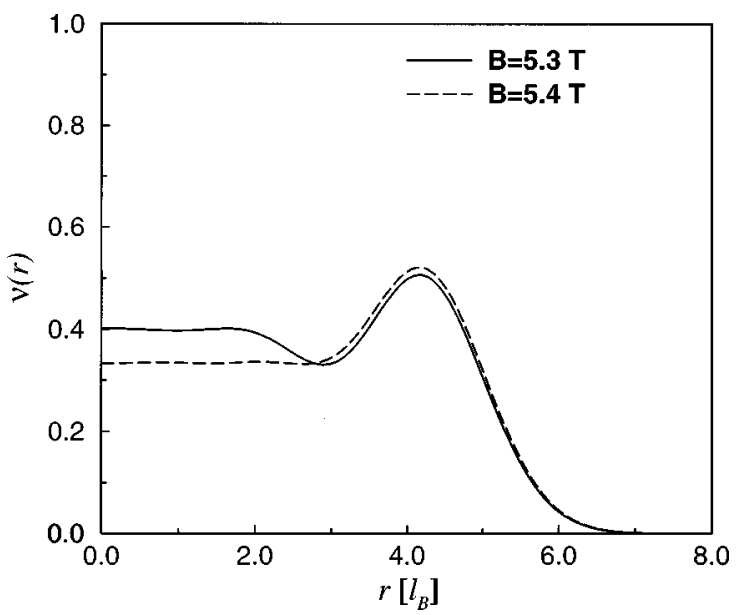

FIG. 2. Local filling factor as a function of radial coordinate for a six-particle system in a parabolic external potential with $\hbar \Omega=2.0 \mathrm{meV}$. Here, the Fano-Ortolani exchange-correlation energy (Ref. 25) was used. The transition to a $1 / 3$ droplet occurs between $B=5.3 \mathrm{~T}$ and $B=5.4 \mathrm{~T}$. The bump in electron charge at the edge of the system is characteristic of systems with a not too soft confining potential. tions used the full Coulomb interaction. Despite these obstacles, the agreement between our ensemble DFT results for small systems and the numerical diagonalizations ${ }^{6}$ are quite good. We also did these calculations at a finite temperature of $100 \mathrm{mK}\left[k_{B} T \sim 1 \times 10^{-3} e^{2} /\left(\epsilon_{0} l_{B}\right)\right]$, which improves the convergence of these small particle systems. We are presently working on extending the Fano-Ortolani interpolation to include $\nu \geqslant 1$ and several Landau levels.

\section{SPIN-TEXTURED EDGE RECONSTRUCTION OF THE MAXIMUM DENSITY DROPLET}

It was once widely thought that that $\nu=1$ is a Fermi liquid in the sense that the elementary excitations are well described by single-particle excitations and only renormalized by the interactions. However, recent experiments on highmobility GaAs quantum wells ${ }^{26}$ have provided evidence for the existence of topological charge-spin textures, so-called Skyrmions, near $\nu=1$. These are nontrivial many-body excitations due to electron-electron interactions first predicted to be the low-energy excitations near $\nu=1$ by Sondhi et al. ${ }^{27}$ with the energies about half of those of single-particle spinflip excitations. The fact that Skyrmions are the low-energy excitations near $\nu=1$ (and also possibly near $\nu=1 / 3$ ) raises the possibility of spin-textured edge reconstruction of the maximum density droplet. Therefore, inclusion of the spin degree of freedom may be essential in the study of inhomogeneous systems. Indeed, Hartree-Fock and effective-field theoretical calculations have shown that for a soft confining potential, the edge of an infinite Hall bar at $\nu=1$ becomes unstable to spin-textured reconstruction for weak Zeeman coupling, while stronger Zeeman coupling yields a spinpolarized reconstruction. ${ }^{28,29}$

Motivated by these ideas, we have generalized our ensemble DFT approach to include spin degrees of freedom within the local spin-density approximation (LSDA). The Hohenberg-Kohn theorem formally ensures that every property, including the spin density or polarization, can be obtained from the ground-state density. However, practical LDA calculations of systems with spontaneously broken symmetries, such as spin rotation symmetry, typically are much improved if the order parameter of the broken symmetry, e.g., spin density or polarization, is explicitly included by construction. In particular, the broken symmetry may not otherwise be obtained accurately from the LDA.

\section{A. Ensemble spin-density-functional theory}

An exact treatment of the spins, in general, requires ${ }^{30}$ the replacement of the charge density $n(\mathbf{r})$ by the single-particle density matrix $\rho_{\sigma \sigma^{\prime}}(\mathbf{r})=\left\langle 0\left|\hat{\psi}_{\sigma}^{+}(\mathbf{r}) \hat{\psi}_{\sigma^{\prime}}(\mathbf{r})\right| 0\right\rangle$. Here, $\hat{\psi}_{\sigma}^{+}(\mathbf{r})$ and $\hat{\psi}_{\sigma}(\mathbf{r})$ are the usual field operators corresponding to the annihilation and creation of an electron with spin $\sigma$ at $\mathbf{r}$, and $|0\rangle$ is the ground state of the system. With a constant magnetic field applied in the $z$ direction, the $\hat{z}$ component of the total spin angular momentum is a constant of the motion and it is convenient to assume that the magnetization density only has a $\hat{z}$ component. Under this assumption, the singleparticle density matrix can be taken to be diagonal, $\rho_{\sigma \sigma^{\prime}}(\mathbf{r})=\rho_{\sigma \sigma^{\prime}}(\mathbf{r}) \delta_{\sigma \sigma^{\prime}}$. In this case the energy functional of Eqs. (1) and (2) is modified to ${ }^{32}$ 


$$
E\left[n_{\uparrow}, n_{\downarrow}\right]=F\left[n_{\uparrow}, n_{\downarrow}\right]+\int d \mathbf{r} n(\mathbf{r}) V_{\mathrm{ext}}(\mathbf{r}),
$$

where $F\left[n_{\uparrow}, n_{\downarrow}\right]=\inf _{\Psi \rightarrow n_{\uparrow}, n}\left\langle\Psi\left|\hat{T}+\hat{E}_{\mathrm{Z}}+\hat{V}_{\text {ee }}\right| \Psi\right\rangle$ and $\hat{E}_{\mathrm{Z}}$ is the Zeeman energy. The infimum is over $\Psi$ yielding fixed densities $n_{\sigma}(\mathbf{r})$. The LSDA is then given by

$$
E_{\mathrm{xc}}^{\mathrm{LSDA}}\left[n_{\uparrow}, n_{\downarrow}\right]=\int d \mathbf{r} n(\mathbf{r}) \epsilon_{\mathrm{xc}}\left[n_{\uparrow}(\mathbf{r}), n_{\downarrow}(\mathbf{r})\right],
$$

where $\epsilon_{\mathrm{xc}}\left[n_{\uparrow}, n_{\downarrow}\right]$ is the exchange-correlation energy per particle in a homogeneous system with up- and down-spin densities $n_{\uparrow}$ and $n_{\downarrow}$, respectively. In spite of the LSDA being justified only in the limit of small spatial variations of the electron density, this approximation has been surprisingly successful in describing the properties of inhomogeneous atomic, molecular, and solid-state systems. ${ }^{33}$ This scheme correctly predicted, for example, ferromagnetism in $\mathrm{Fe}, \mathrm{Co}$, and $\mathrm{Ni}$ among the transition metals. ${ }^{33}$ Moreover, the selfinteraction-corrected LSDA was successfully applied to some strongly correlated systems such as the transition-metal oxides and a Hubbard model representing a $\mathrm{CuO}_{2}$ layer in the cuprate superconductors. ${ }^{34}$

For a parabolic dot, the variational principle applied to the $\mathrm{KS}$ functional [Eq. (11)] yields two sets of KS equations for spin-up and spin-down electrons,

$$
\left[T+V_{s, \sigma}(r, B)\right] \varphi_{m n, \sigma}(r)=\varepsilon_{m n, \sigma} \varphi_{m n, \sigma}(r),
$$

where

$$
V_{s, \sigma}(r, B)=\sigma g^{\star} \mu_{0} B+V_{\mathrm{ext}}(r)+V_{\mathrm{H}}(r)+V_{\mathrm{xc}, \sigma}(r, B)
$$

is an effective potential for the auxiliary noninteracting system. In the LSDA the exchange-correlation potentials are

$$
V_{\mathrm{xc}, \sigma}(r, B)=\left.\frac{\partial}{\partial n_{\sigma}}\left(n \epsilon_{\mathrm{xc}}\left[n_{\uparrow}, n_{\downarrow}, B\right]\right)\right|_{n_{\sigma}=n_{\sigma}(r)} .
$$

The parametric dependence on the magnetic field $B$ can be incorporated by using spin filling factors $\nu_{\sigma}=2 \pi l_{B}^{2} n_{\sigma}$ as variables instead of spin densities $n_{\sigma}$. To make connection with the spin-polarized case we first transform the spin filling factors $\nu_{\sigma}$ to total filling factor $\nu$ and spin polarization $\xi$ :

$$
\begin{gathered}
\nu=\nu_{\uparrow}+\nu_{\downarrow}, \\
\xi=\left(\nu_{\uparrow}-\nu_{\downarrow}\right) /\left(\nu_{\uparrow}+\nu_{\downarrow}\right) .
\end{gathered}
$$

The exchange-correlation potentials [Eq. (15)] then become

$$
\begin{aligned}
& V_{\mathrm{xc}, \uparrow}=\frac{\partial}{\partial \nu}\left(\nu \epsilon_{\mathrm{xc}}\right)+(1-\xi) \frac{\partial}{\partial \xi} \epsilon_{\mathrm{xc}}, \\
& V_{\mathrm{xc}, \downarrow}=\frac{\partial}{\partial \nu}\left(\nu \epsilon_{\mathrm{xc}}\right)-(1+\xi) \frac{\partial}{\partial \xi} \epsilon_{\mathrm{xc}}
\end{aligned}
$$

where the exchange-correlation energy per particle in a homogeneous system with a filling factor $\nu$ and polarization $\xi$, i.e., $\epsilon_{\mathrm{xc}} \equiv \epsilon_{\mathrm{xc}}(\nu, \xi)$, has to be approximated.

\section{B. Constructing $V_{\mathrm{xc}}(\nu, \xi)$}

Our approach in constructing the exchange-correlation potentials $V_{\mathrm{xc}}(\nu, \xi)$ is to first construct the exchangecorrelation energy per particle for a spin-polarized homogeneous system $\epsilon_{\mathrm{xc}}$, and then to add interpolation functions to obtain the exchange-correlation energy at arbitrary polarization $\xi$. We choose as exchange-correlation energy per particle in a homogeneous spin-polarized system,

$$
\epsilon_{\mathrm{xc}}(\nu)=\epsilon_{\mathrm{xc}}^{\mathrm{LWM}}(\nu)+\epsilon_{\mathrm{xc}}^{C}(\nu) .
$$

The first term is a smooth interpolation formula of Levesque, Weis, and MacDonald ${ }^{24}$

$$
\begin{aligned}
\epsilon_{\mathrm{xc}}^{\mathrm{LWM}}(\nu)= & \int_{0}^{\infty} d r r\left(\frac{e^{2}}{r \epsilon_{0} l_{B}}\right)\left[g_{\nu}(r)-1\right] \\
\simeq & -0.782133 \sqrt{\nu}\left(1-0.211 \nu^{0.74}+0.012 \nu^{1.7}\right) \\
& \times\left(e^{2} / \epsilon_{0} l_{B}\right),
\end{aligned}
$$

for the ground-state energy obtained by evaluating the pair correlation functions $g_{\nu}(r)$ at certain fillings $\nu<\frac{1}{2}$ for about 256 particles using very accurate Monte Carlo methods. The second term in Eq. $(18), \epsilon_{\mathrm{xc}}^{C}(\nu)$, contains the cusps in the ground-state energy that cause the FQHE. The discontinuity in the slope of $\epsilon_{\mathrm{xc}}^{C}(\nu)$ near certain "magic'" filling factors $\nu^{\star}=p / q$ is related to the chemical potential gap $\Delta \mu=q\left(\left|\Delta_{p}\right|+\left|\Delta_{h}\right|\right)$. Here $\Delta_{p, h}$ are the quasiparticle (hole) creation energies $^{31}$ at $\nu=\nu^{\star}$. In our calculations, we restrict ourselves to include only the cusps at $\nu=1 / 3,2 / 5,3 / 5$, and $\nu=2 / 3$, which are the strongest fractions. (See the Appendix for a detailed description of our expression for $\epsilon_{\mathrm{xc}}^{C}$.) Substituting Eq. (18) into Eq. (6) gives the exchange-correlation potential as a function of filling factor $V_{\mathrm{xc}}(\nu)$ for a spinpolarized system. This potential is depicted in Fig. 3 as $V_{\mathrm{xc}, \uparrow}(\nu, \xi=1)$.

The question is then how to obtain a reasonable interpolation formula for $\epsilon_{\mathrm{xc}}$ between spin-polarized $(\xi=1)$ and spin-unpolarized ( $\xi=0)$ two-dimensional (2D) electron liquids for a fixed $\nu$ in a strong magnetic field. We have constructed a first approximation, as we will now explain. The result is fairly complicated (a piecewise interpolation in two variables among various numerically and analytically obtained results) and is not well represented as a single formula. A subroutine calculating $V_{\mathrm{xc}}(\nu, \xi)$ can be obtained from the authors.

In what follows $\mathrm{x}(\mathrm{c})$ as a subscript denotes exchange (correlation) respectively. We decompose the exchangecorrelation energy $E_{\mathrm{xc}}$ into exchange $E_{\mathrm{x}}$ and correlation $E_{\mathrm{c}}$ energies. Since the exchange interaction only acts between parallel spins, we have

$$
E_{\mathrm{x}}\left[\nu_{\uparrow}, \nu_{\downarrow}\right]=\frac{1}{2} E_{\mathrm{x}}\left[\nu_{\uparrow}, \nu_{\uparrow}\right]+\frac{1}{2} E_{\mathrm{x}}\left[\nu_{\downarrow}, \nu_{\downarrow}\right]
$$

Moreover, it follows from dimensional analysis that the exchange energy must scale as density (filling factor) to the $3 / 2$ power in a $2 \mathrm{D}$ electron gas. Following Oliver and Perdew, ${ }^{35}$ we can then write 


$$
E_{\mathrm{x}} \sim \int d^{2} r\left[\nu_{\uparrow}^{3 / 2}(r)+\nu_{\downarrow}^{3 / 2}(r)\right] .
$$

We also have from Eqs. (16) $\nu_{\uparrow}=\frac{1}{2} \nu(1-\xi), \nu_{\downarrow}=\frac{1}{2} \nu(1+\xi)$. Equation (21) can then be rewritten as

$$
E_{\mathrm{x}} \sim \int d^{2} r \nu^{3 / 2}\left[(1+\xi)^{3 / 2}+(1-\xi)^{3 / 2}\right] .
$$

Since in the local-density approximation for the exchange energy $E_{\mathrm{x}}=\int d^{2} r \nu \epsilon_{\mathrm{x}}(\nu, \xi)$ we are then led to the form

$$
\begin{aligned}
\epsilon_{\mathrm{x}}(\nu, \xi) & =\epsilon_{\mathrm{x}}(\nu, \xi=1)+\left(\epsilon_{\mathrm{x}}(\nu, \xi=0)-\epsilon_{\mathrm{x}}(\nu, \xi=1)\right) f(\xi) \\
& \equiv \epsilon_{\mathrm{x}}(\nu, \xi=1)+\Delta \epsilon_{\mathrm{x}}(\nu, \xi),
\end{aligned}
$$

where the function

$$
f(\xi)=\frac{(1+\xi)^{3 / 2}+(1-\xi)^{3 / 2}-2 \sqrt{2}}{2-2 \sqrt{2}}
$$

is an interpolation function between the two extreme cases $\xi=0$ and $\xi=1$ with $f(0)=1$ and $f(1)=0$. Although the analogous simple closed form for the correlation energy $\epsilon_{\mathrm{c}}(\nu, \xi)$ is not available, it can be always be written as $\epsilon_{\mathrm{c}}(\nu, \xi)=\epsilon_{\mathrm{c}}(\nu, \xi=1)+\Delta \epsilon_{\mathrm{c}}(\nu, \xi)$. So, as a first approximation we will use the form of Eq. (23) for the smooth part of the correlation energy $\epsilon_{\mathrm{c}}$, too (leaving the cusps aside for the moment), with the same interpolation function $f(\xi)$, as was suggested first by von Barth and Hedin. ${ }^{30}$ Denoting the smooth part of the exchange-correlation energy per particle by $\epsilon_{\mathrm{xc}}^{\mathrm{s}}$ we can then write

$$
\begin{aligned}
\boldsymbol{\epsilon}_{\mathrm{xc}}^{\mathrm{s}}(\nu, \xi) & =\boldsymbol{\epsilon}_{\mathrm{xc}}^{\mathrm{s}}(\nu, \xi=1)+\left[\epsilon_{\mathrm{xc}}^{\mathrm{s}}(\nu, \xi=0)-\epsilon_{\mathrm{xc}}^{\mathrm{s}}(\nu, \xi=1)\right] f(\xi) \\
& \equiv \epsilon_{\mathrm{xc}}^{\mathrm{s}}(\nu, \xi=1)+\delta \epsilon_{\mathrm{xc}}(\nu) f(\xi) .
\end{aligned}
$$

So far, we have constructed a function $\epsilon_{\mathrm{xc}}^{\mathrm{s}}(\nu, \xi)$ that gives a smooth interpolation for the exchange-correlation energy for any value of $\nu$ and $\xi$. What is left is to add the cusps to this function. We already have a good approximation for these at $\xi=1$. We now need to extend this approximation to arbitrary values of $\xi$. Very little is known about the cusps, i.e., the energy gaps, for arbitrary polarizations. It is known that there is a gap for unpolarized systems at fillings $\nu=2 / 5, \nu=3 / 5$, and $\nu=2 / 3$. The gap, and thus the cusps, occur at very special "magic" configurations at which the system can take advantage of a particularly low correlation energy. Therefore, it seems plausible that for a given value of $\nu$, say, $\nu=2 / 5$, there cannot be an energy gap for any value of $\xi$ between 0 and 1 . In order to incorporate this assumption into a usable approximation, we interpolate our cusp energy constructed for polarized systems, $\epsilon_{\mathrm{xc}}^{\mathrm{C}}(\nu)$, to arbitrary polarizations by multiplying it by a function $g(\xi)$ that is unity at $\xi=0$ and $\xi=1$ with zero derivative at these points, vanishes away from these values of polarization, and is symmetric about $\xi=1 / 2$. All together, then, we have

$$
\boldsymbol{\epsilon}_{\mathrm{xc}}(\nu, \xi)=\boldsymbol{\epsilon}_{\mathrm{xc}}^{\mathrm{LWM}}(\nu)+\delta \boldsymbol{\epsilon}_{\mathrm{xc}}(\nu) f(\xi)+\boldsymbol{\epsilon}_{\mathrm{xc}}^{\mathrm{C}}(\nu) g(\xi) .
$$

Specifically, we chose

$$
g(\xi)=\left[4 \xi^{2}-1\right]^{2}\left[27-\xi^{2}\left(40-16 \xi^{2}\right)\right] / 27,
$$

which is the only polynomial in $\xi$ satisfying the above constraints. Near $\nu=1$ (where there is no cusp in the total exchange-correlation energy), the sign of the function $\delta \epsilon_{\mathrm{xc}}(\nu)$ will then determine the spin-polarized (ferromagnetic) or spin-unpolarized (paramagnetic) ground state of the infinite electron liquid (neglecting the Zeeman splitting). Indeed, substitution of Eq. (26) into Eq. (17) gives

$$
\begin{aligned}
\Delta V_{\mathrm{xc}} \equiv & V_{\mathrm{xc}, \uparrow}-V_{\mathrm{xc}, \downarrow}=2 \frac{\partial}{\partial \xi} \epsilon_{\mathrm{xc}}=2 \delta \epsilon_{\mathrm{xc}}(\nu) f^{\prime}(\xi) \\
& +2 \epsilon_{\mathrm{xc}}^{\mathrm{C}}(\nu) g^{\prime}(\xi) .
\end{aligned}
$$

The last term in this expression may be ignored near $\nu=1$. We would thus expect a spin-polarized ground state if $\delta \epsilon_{\mathrm{xc}}(\nu)>0$ because $f^{\prime}(\xi)<0$ for all $0 \leqslant \xi \leqslant 1$, so in this case the inequality $V_{\mathrm{xc}, \uparrow}<V_{\mathrm{xc}, \downarrow}$ holds. Otherwise we would expect a spin-unpolarized state. In contrast, numerical diagonalizations suggest that, at some fillings, the homogeneous ground state is partially polarized. This cannot be obtained by our simple model of the exchange-correlation energy with a monotonic interpolation function $f(\xi)$. Nevertheless, the simple model of Eq. (25) allows us to capture the essential physics of the spin-unpolarized edge reconstruction of the quantum dot in reasonable agreement with other methods, as we will show.

In order to obtain the function $\delta \epsilon_{\mathrm{xc}}(\nu)$ in Eq. (26) we start by calculating the energy differences between spin-polarized and -unpolarized states using small-system numerical diagonalization data for some filling fractions obtained from Chakraborty and Zhang. ${ }^{36}$ The value for the ground-state energy of a $\nu=1$ unpolarized system is not available, but a reasonable approximation is to take $\epsilon_{\mathrm{xc}}(\nu=1, \xi=0)=$ $\epsilon_{\mathrm{xc}}(\nu=1 / 2, \quad \xi=1)=-0.469\left(e^{2} / \epsilon_{0} l_{B}\right)$, implying that the spin-up and spin-down components are uncorrelated. (This most likely overestimates the energy.) The ground-state energy of a $\nu=1$ polarized system $\epsilon_{\mathrm{xc}}(\nu=1, \xi=1)$ $=-0.6265\left(e^{2} / \epsilon_{0} l_{B}\right)$. Therefore we have $\delta \epsilon_{\mathrm{xc}}(\nu=1)$ $=0.1575\left(e^{2} / \epsilon_{0} l_{B}\right)$. To complete the numerical parameterization of the exchange-correlation functional, we then perform a spline fit to obtain the function $\delta \epsilon_{\mathrm{xc}}(\nu)$. We have plotted the exchange-correlation potentials $V_{\mathrm{xc}, \sigma}$ as a function of a filling factor $\nu$ at $\xi=1$ and $\xi=0$ in Fig. 3. We see that at $\xi=1$ the difference between exchange-correlation potentials for spin-up and spin-down electrons $\Delta V_{\text {xc }}$ [Eq. (28)] is changing sign from negative to positive while the filling factor $\nu$ is decreasing from $\nu=1$ to $\nu=2 / 3$. Ignoring the Zeeman splitting and the cusps, the ground state of an infinite electron liquid would change from spin polarized to spin unpolarized. To estimate the possibility of having a spinunpolarized state above filling $2 / 3$ with the inclusion of the Zeeman splitting, we have to compare the dimensionless Zeeman energy $\widetilde{g}=g^{*} \mu_{B} B /\left[e^{2} /\left(\epsilon_{0} l_{B}\right)\right]$ with the difference $\Delta V_{\mathrm{xc}}$ [Eq. (28)] at this filling $\Delta V_{\mathrm{xc}}(\nu=2 / 3, \xi=1) \approx$ $0.05\left(e^{2} / \epsilon_{0} l_{B}\right)$. This value is larger then the Zeeman splitting for GaAs $\widetilde{g} \approx 0.02$. Therefore, the ground state of a GaAsbased homogeneous system is a spin-unpolarized state at and just above filling factor $2 / 3$. In an inhomogeneous system, in addition to exchange-correlation potential and Zeeman energy, there are also the Hartree interaction of the 2D electrons and the external potential that confines them. Hence, 


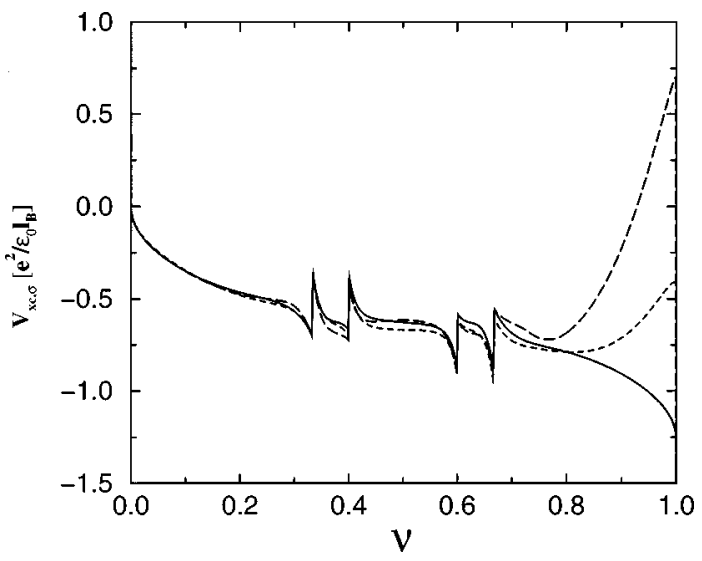

FIG. 3. The exchange-correlation potentials $V_{\mathrm{xc}, \sigma}$ as a function of a filling factor $\nu$ at $\xi=1$ and $\xi=0$ in units of $e^{2} /\left(\epsilon_{0} l_{B}\right)$. The solid line indicates $V_{\mathrm{xc}, \uparrow}$ and short-dashed line corresponds to $V_{\mathrm{xc}, \downarrow}$ at $\xi=1$. According to Eqs. (28) and (24), the exchange-correlation potentials $V_{\mathrm{xc}, \sigma}$ coincide at $\xi=0\left[\right.$ since $\left.f^{\prime}(0)=0\right]$ and are shown by the long-dashed line. The increase in $V_{\mathrm{xc}}$ as functions of $\nu$ at a FQHE filling factors occurs over a range of a filling factor of 0.002 .

even in our simple approximation, it is possible to have not only polarized and unpolarized states, but also a partially polarized state in an inhomogeneous system such as a quantum dot.

\section{Spin textures on edges}

We have reinvestigated the stability of the MDD using our LSDA ensemble DFT. Early numerical diagonalizations of small systems ${ }^{6}$ indicated that the initial reconstruction of the MDD was to spin-polarized states only for unphysically large Zeeman energies. With $g^{*}$ in the range of physical values, the reconstruction of the polarized MDD was found to be to a partially polarized state. More recent work by Karlhede et al. ${ }^{28}$ and by Franco and Brey ${ }^{29}$ has also investigated the edge reconstruction of a Hall bar using the Hartree-Fock approximation. Karlhede et al. found that, for physical values of $g^{*}$, the reconstruction of a spin-polarized Hall bar at $\nu=1$ (the analogy in a rectangular geometry of an MDD) was to a spin-textured edge. These works then suggest that in order to obtain a correct picture of edge reconstruction, the spin degree of freedom has to be included. This is an important point, especially in view of the fact that there are now very sensitive experimental probes of QHE edges. However, until now the interpretations of experimental results have relied on the picture by Chklovskii, Shklovskii, and Glazman ${ }^{9}$ for fully spin-polarized edges, which then may have to be modified to take the electron spins into account. Our motivation to reinvestigate the stability of the MDD is to demonstrate that our DFT approach yields results in agreement with previous work using different methods that are limited in their applicability, and thus that our DFT approach can be used to study spin structures in general inhomogeneous QHE systems. We expect this latter point to be an important area of future research.

As a model system, we chose a parabolic dot with the same parameters in Sec. III. The focus of the investigation was the nature of the initial instability of the MDD as the effective confinement strength

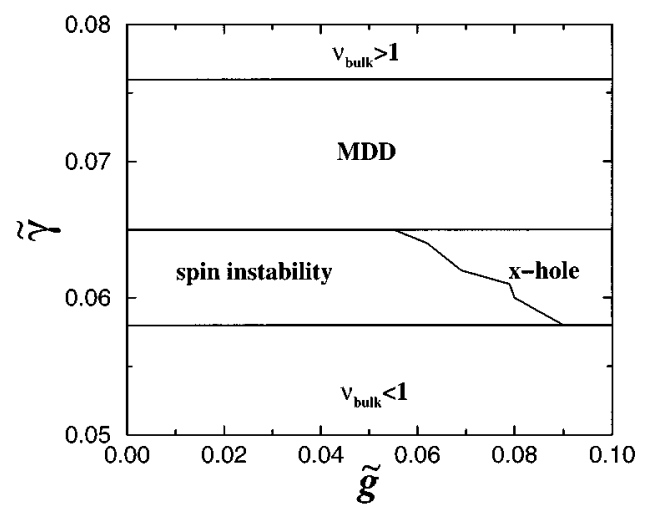

FIG. 4. Phase diagram of the edge reconstruction of a parabolic quantum dot in the $(\widetilde{g}, \widetilde{\gamma})$ plane for $N=38$ electrons. Here, the confining potential has a strength given by $\hbar \Omega=1.6 \mathrm{meV}$. For $\tilde{\gamma}>0.065$, the system forms a maximum density droplet for all values of the Zeeman coupling $\widetilde{g}$. For values of the Zeeman coupling $\widetilde{g}$ larger than a critical value $\widetilde{g_{c}}$, the maximum density droplet undergoes an initial reconstruction to a spin-polarized exchange hole as the confinement strength $\tilde{\gamma}$ is decreased, while for $\widetilde{g}<\widetilde{g}_{c}$ the maximum density droplet has a spin-structured instability with decreasing $\widetilde{\gamma}$. In these calculations, $\widetilde{g_{c}} \approx 0.055$.

$$
\tilde{\gamma}=\frac{m^{*} \Omega^{2} l_{B}^{2}}{\left(e^{2} / \epsilon_{0} l_{B}\right)}
$$

is decreased (by increasing the magnetic-field strength) as a function of the Zeeman coupling. The Zeeman coupling is most conveniently expressed in a dimensionless form as the ratio of the Zeeman energy to the Coulomb energy,

$$
\widetilde{g}=\frac{g^{*} \mu_{B} B}{\left(e^{2} / \epsilon_{0} l_{B}\right)} .
$$

We find that for large enough values of $\widetilde{g}$, the instability is to the fully spin-polarized "exchange hole," in which a lump of charge is expelled at the edge to decrease the Hartree repulsion while still taking advantage of the short-range attractive exchange interaction. However, as $\tilde{g}$ is decreased below a certain value $\widetilde{g_{c}}$, the instability is from the spinpolarized MDD to a state in which the edge is partially polarized. The value $\widetilde{g_{c}}$ at which minority spins first appear at the edge, is for this system $\widetilde{g}_{c} \approx 0.055$. We can then plot a phase diagram for the parabolic dot near the MDD region in the $\tilde{g}-\tilde{\gamma}$ plane. ${ }^{6,28}$ This phase diagram is depicted in Fig. 4. For values of $\tilde{\gamma}$ less than approximately 0.076 , the system forms an MDD (for $\tilde{\gamma} \gtrsim 0.076$ there is appreciable occupation of states in the higher Landau levels). At $\tilde{\gamma} \approx 0.065$ an instability to higher total angular momentum occurs and the MDD reconstructs. For $g>\widetilde{g} \approx 0.055$ the reconstruction is to the totally polarized exchange hole (this region is marked " $x$ hole" in Fig. 4). For fixed $\widetilde{g}$ such that $\widetilde{g_{c}} \leqslant \widetilde{g} \leq 0.085$ the system undergoes an additional instability as $\widetilde{\gamma}$ is increased, in which minority-spin population appears at the edge. For $\tilde{g}<\widetilde{g}_{c}$ the initial instability of the MDD is always to a state with a partially polarized edge. Finally, as $\widetilde{\gamma}$ is decreased below approximately 0.058 , the system will undergo further reconstructions, the nature of which depends on the value of $\widetilde{g}$. We have not here studied in detail the state reached after these reconstructions. The value of $\widetilde{g_{c}} \approx 0.055$ separating the 
spin-polarized and spin-structured instabilities is in reasonable agreement with the value $\widetilde{g_{c}} \approx 0.03$ found (for much smaller systems) using numerical diagonalization. ${ }^{6}$ A phase diagram analogous to ours was obtained by Hartree-Fock calculations by Karlhede et al. ${ }^{28}$ Their value of the critical Zeeman splitting $\widetilde{g_{c}}=0.169$ is about three times larger then $\widetilde{g_{c}} \approx 0.055$ from our phase diagram. In contrast, Franco and Brey ${ }^{29}$ obtained for a Hall bar geometry a value of $\widetilde{g_{c}} \approx 0.008$ below which the edge is unstable with respect to spintextured edge reconstruction. Note that for the value of the confinement strength used in our calculations, $\hbar \Omega=1.6 \mathrm{meV}$, we have $\widetilde{g} \approx 0.01$ at a magnetic field strength of $2.8 \mathrm{~T}$, at which the instability of the MDD occurs. This means that for physical values of $g^{*}$, the instability will in these calculations always occur to a state with a partially polarized edge. Future work will be extended to include charge modulations along the direction of the edge. ${ }^{29}$

Within the LSDA it is easy to understand why the edge's spin state changes as the Zeeman coupling $\widetilde{g}$ changes. As is seen in Fig. 3, $V_{\mathrm{xc}, \downarrow}<V_{\mathrm{xc}, \uparrow}$ for $\nu \leqq 0.8$ and $\xi=1$, so for small filling factors $(\nu \leq 0.8)$ the exchange-correlation energy destabilizes polarized densities in favor of unpolarized densities. The Zeeman coupling, conversely, favors polarized densities (majority spins). At the edge as the density decreases there is then a competition between exchange-correlation and Zeeman energies. For large enough Zeeman coupling $\left(\tilde{g}>\tilde{g}_{c}\right)$, it is energetically favorable for the edge to remain spin polarized. For small Zeeman coupling $\left(\widetilde{g}<\widetilde{g}_{c}\right)$, the exchange-correlation effects dominate the Zeeman coupling, and a minority-spin population appears at the edge. This is depicted in Fig. 5, which shows an example with $\tilde{g}=0.014$ and $\tilde{\gamma}=0.063$. These are values such that the system has just undergone a reconstruction to a partially polarized edge. In the following the majority-spin direction is "up" and the minority "down." Figure 5(a) shows a lump of charge that has been expelled in the reconstruction (solid line), but this expelled charge is partially polarized (dashed line). This is clearly depicted in Fig. 5(b), which depicts up- and downspin occupancies. In the region where the expelled charge resides, there are partially occupied down-spin states $(\diamond)$ together with almost fully occupied up-spin states $(+)$. This implies that these up- and down-spin states all have to be degenerate at the Fermi level, as is indeed shown in Fig. 5(c). The behavior of the KS eigenvalues depicted in Fig. 5(c) is generic: for a fixed value of $\tilde{\gamma}$ in the region of the initial MDD instability, the KS eigenvalues of the down-spin states are higher than those for the up-spins states at large values of $\widetilde{g}$. As $\tilde{g}$ is reduced, the difference between the up-spin and down-spin KS eigenvalues decreases in the edge region as the strength of exchange correlation effects increases relative to the Zeeman coupling. At some $\tilde{g}=\widetilde{g_{c}}$, the difference vanishes and both up- and down-spin states become occupied at the edge. We would also like to point out that because many KS orbitals are degenerate at the Fermi energy, an ensemble DFT approach has to be used.

\section{SUMMARY AND CONCLUSIONS}

We have developed a spin-ensemble density-functional approach and used it to study inhomogeneous quantum Hall systems in the integer and fractional Hall regimes. For spin-
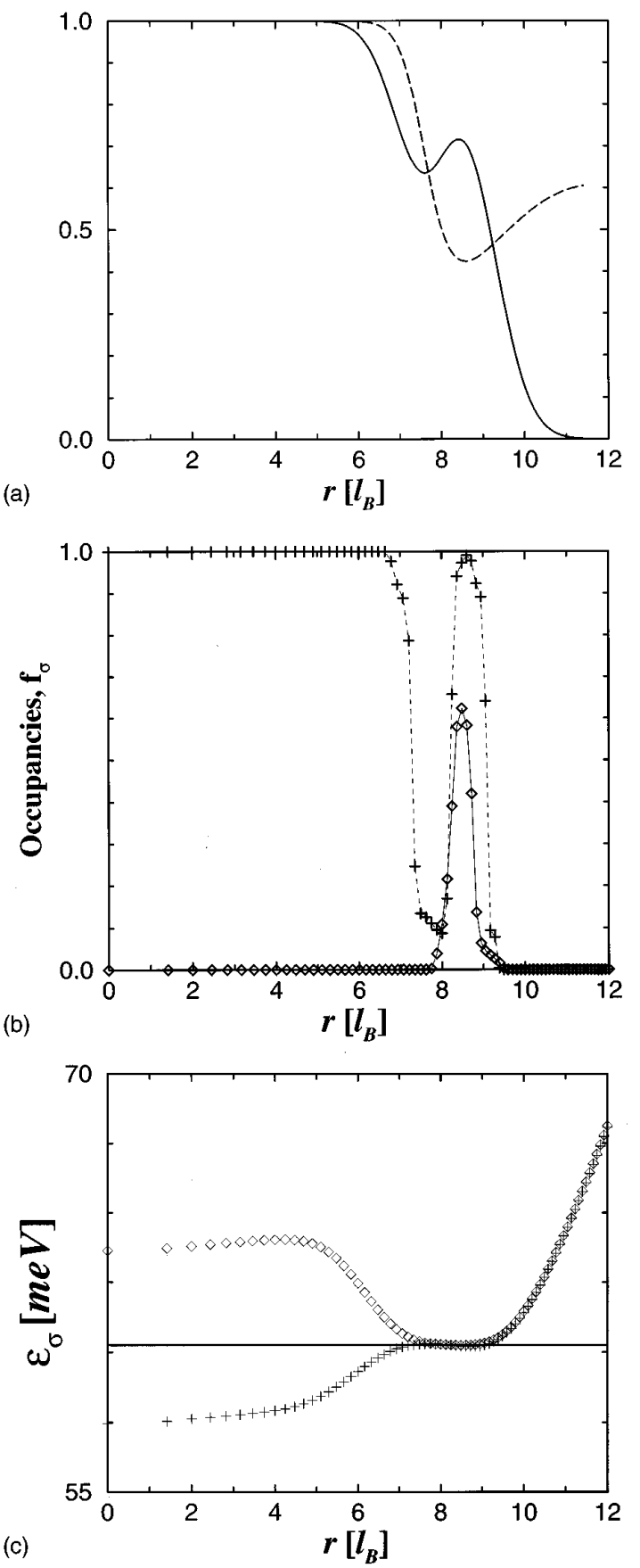

FIG. 5. Spin structured instability at the edge of a quantum dot for a Zeeman splitting $\widetilde{g}=0.014$, magnetic field $B=3.05 \mathrm{~T}$, and $N=38$. The external potential is characterized by $\hbar \Omega=1.6 \mathrm{meV}$ (so that the dimensionless strength of the confinement is $\tilde{\gamma}=0.063$ ). (a) The solid line depicts the total local filling factor $\nu(r)$ vs radial coordinate $r$, and the dashed line depicts the polarization. (b) The occupancies of the KS states $\psi_{m 0, \sigma}(r)$ are depicted vs orbital center coordinate $r_{m}=\sqrt{2 m} l_{B}$ with + for majority $(\uparrow)$ spin occupancies, and $\diamond$ for minority $(\downarrow)$ spin occupancies. At the instability of the maximum density droplet for this value of $\widetilde{g}$, there is a minorityspin population at the edge of the system. (c) Eigenvalues of the two lowest-Landau-level KS orbitals, with + depicting eigenvalues of the majority-spin orbitals, and $\diamond$ depicting the eigenvalues of the minority-spin orbitals. The chemical potential is indicated by the solid line. At the edge the filling factor takes fractional values, and the KS eigenvalues are here degenerate and equal to the Fermi energy $\varepsilon_{F}$, in agreement with the general theory of Sec. II. 
polarized systems, our approach gives results in excellent agreement with numerical diagonalizations, Hartree-Fock calculations, and semiclassical calculations. Note that while all of these latter approaches have limited regions of applicability, such as small systems, systems near $\nu=1$, or the semiclassical limit, we have here demonstrated that our ensemble density-functional approach spans all these regions, which makes it a useful approach to general inhomogeneous quantum Hall systems.

We have generalized the ensemble DFT to include spin degrees of freedom within a simple local-spin-density approximation, and applied this generalization to a quantum dot. Our results show that for small, but physical, Zeeman energies, $\widetilde{g}<\widetilde{g_{c}}$, the maximum density droplet is unstable with respect to spin-textured edge reconstructions as the magnetic field increased. At larger Zeeman splittings, $\widetilde{g}>\widetilde{g}_{c}$, the maximum density droplet is unstable with respect to spin-polarized edge reconstructions. Our value of $\widetilde{g_{c}}$ is in good agreement with that obtained from numerical diagonalization studies. ${ }^{6}$ Hartree-Fock calculations for an infinite Hall bar by Karlhede et al. ${ }^{28}$ give a phase diagram qualitatively analogous to ours. However, Hartree-Fock calculations are limited to $\nu \approx 1$, while our ensemble DFT is, in principle, applicable to general fractional quantum Hall systems, e.g. droplets at $\nu=\frac{1}{3}$. The accuracy of our approach depends on obtaining good estimates of the exchangecorrelation energy as a function of both electron density and spin polarization for homogeneous fractional quantum Hall systems. Work is currently in progress to improve these estimates. Finally, the spin ensemble DFT used here cannot be used to study spin-charge textures (Skyrmions), in which the spin polarization rotates smoothly in space. Work is currently under way to generalize our spin DFT to include such charge-spin textures.

\section{ACKNOWLEDGMENTS}

The authors would like to thank M. Ferconi, M. Geller, and G. Vignale for helpful discussions and for sharing their results prior to publication, K. Burke and E. K. U Gross for useful comments about the DFT, M. Levy for a discussion about Ref. 15, and J. M. Kinaret for help with the LSDA. O.H. would like to thank Chalmers Institute of Technology, where part of the work was done. This work was supported by the NSF through Grants Nos. DMR93-01433 and DMR96-32141.

\section{APPENDIX}

We will construct the cusp part of the exchangecorrelation energy, $\epsilon_{\mathrm{xc}}^{C}(\nu)$ for a spin-polarized system by first considering $\nu<1 / 2$, and then use electron-hole symmetry to obtain the form for $1 / 2<\nu<1$. Finally, for $\nu>1$ we assume $\epsilon_{\mathrm{xc}}^{C}(\nu)=\epsilon_{\mathrm{xc}}^{C}(1-\nu)$.

For spin-polarized systems in the lowest Landau level, we write $\epsilon_{\mathrm{xc}}(\nu)=\epsilon_{\mathrm{xc}}^{s}(\nu)+\epsilon_{\mathrm{xc}}^{C}(\nu)$, where $\epsilon_{\mathrm{xc}}^{s}(\nu)$ is given by a smooth interpolation, such as the Levesque-WeissMacDonald formula ${ }^{24}$ (although this one does not obey strict particle-hole symmetry in the lowest Landau level), or the Fano-Ortolani formula. ${ }^{25}$ Particle-hole symmetry yields for the total exchange-correlation energy

$$
\nu\left[\epsilon_{\mathrm{xc}}(\nu)-\epsilon_{\mathrm{xc}}(1)\right]=[1-\nu]\left[\epsilon_{\mathrm{xc}}(1-\nu)-\epsilon_{\mathrm{xc}}(1)\right],
$$

from which we obtain

$$
\nu \epsilon_{\mathrm{xc}}^{s}(\nu)=\nu^{*} \epsilon_{\mathrm{xc}}^{s}\left(\nu^{*}\right)+\left(1-2 \nu^{*}\right) \epsilon_{\mathrm{xc}}(1),
$$

with $\nu^{*} \equiv 1-\nu$. This means that

$$
\nu \epsilon_{\mathrm{xc}}^{C}(\nu)=\nu^{*} \epsilon_{\mathrm{xc}}^{C}\left(\nu^{*}\right) .
$$

We define

$$
g(\nu) \equiv \nu \epsilon_{\mathrm{xc}}^{C}(\nu) .
$$

Since the discontinuities in the chemical potential at fractional QHE fillings $p / q$ is a relation for $d\left[\nu \epsilon_{\mathrm{xc}}(\nu)\right] / d \nu$, it is easier to work with $g(\nu)$ than with $\epsilon_{\mathrm{xc}}^{C}(\nu)$. Then particlehole symmetry implies that

$$
\frac{d g}{d \nu}=-\frac{d g\left(\nu^{*}\right)}{d \nu^{*}} .
$$

At fractional QHE fillings $p / q$, we must have

$$
\left.\frac{d}{d \nu} g(\nu)\right|_{\nu=(p / q)^{+}}-\left.\frac{d}{d \nu} g(\nu)\right|_{\nu=(p / q)^{-}}=q\left(\mu_{+}+\mu_{-}\right),
$$

where $\mu_{+}$and $\mu_{-}$are the quasiparticle and quasihole creation energies (defined to be positive), respectively. We construct $g(\nu)$ for $0 \leqslant \nu \leqslant 1 / 2$ to be piecewise smooth, with $g(p / q)=0$ for $p / q$ a fractional QHE filling, and a discontinuity in the derivative given by Eq. (A6).

We only included the cusps at $\nu=1 / 3,2 / 5$, their particlehole conjugates, and the corresponding values at fillings increased by unity. For $\nu<1 / 3$, we make the ansatz

$$
g(\nu)=\alpha \nu q \mu_{-}(p / q)(\nu-p / q)\left[e^{(\nu-p / q)}-g_{0}\right],
$$

with $p / q=1 / 3$ and

$$
\alpha=\frac{q}{p\left(1-g_{0}\right)}
$$

and $g_{0}=\exp (-p / q)$.

For $2 / 5<\nu \leqslant 1 / 2$ we take

$$
g(\nu)=\frac{5}{a} \mu_{+}(2 / 5)\left[1-e^{-a_{0}(\nu-2 / 5)}\right],
$$

with $a_{0}=80$.

Next, for $1 / 3<\nu<2 / 5$ we used a cubic interpolation,

$$
g(\nu)=a(\nu-1 / 3)(\nu-2 / 5)\left(\nu-\nu_{3}\right) .
$$

Fixing the slope of $g(\nu)$ at $(1 / 3)^{+}$and $(2 / 5)^{-}$then yields

$$
a=\frac{3 \mu_{+}(1 / 3)}{(1 / 3-2 / 5)\left(1 / 3-\nu_{3}\right)},
$$

and

$$
\nu_{3}=\frac{5 \mu_{-}(2 / 5) / 3+6 \mu_{+}(1 / 3) / 5}{\mu_{+}(1 / 3)+5 \mu_{-}(2 / 5)} .
$$


Finally, we smooth out the resulting discontinuities in $V_{\mathrm{xc}}$ over an interval $2 \delta$ about the fractional QHE fillings $\nu=p / q$. To do this, we interpolate linearly $g(\nu)$ between its values at $\nu=p / q \pm \delta$, so that $d g(\nu) / d \nu=A+B(\nu-p / q+\delta)$, where $A=g(p / q-\delta) \quad$ and $B=[g(p / q+\delta)-g(p / q-\delta)] /(2 \delta)$.
Simple integration then yields $g(\nu)=A \nu+\frac{1}{2} B \nu^{2}-$ $B \nu(p / q-\delta)+C$, where $C$ is an integration constant given by

$$
C=g(p / q-\delta)-A(p / q-\delta)+\frac{1}{2} B(p / q-\delta)^{2} .
$$

${ }^{1}$ O. Heinonen, M. I. Lubin, and M. D. Johnson, Phys. Rev. Lett. 75, 4110 (1995).

${ }^{2}$ O. Heinonen, M. I. Lubin, and M. D. Johnson, Int. J. Quantum Chem. Quantum Chem. Symp. 30, 231 (1996).

${ }^{3}$ O. Heinonen, M. I. Lubin, and M. D. Johnson, in Electronic Density Functional Theory: Recent Progress and New Directions, edited by J. F. Dobson, G. Vignale, and M. P. Das (Plenum, New York, 1997).

${ }^{4}$ K. v. Klitzing, G. Dorda, and M. Pepper, Phys. Rev. Lett. 45, 494 (1980); D. C. Tsui, H. L. Stormer, and A. C. Gossard, ibid. 48, 1559 (1990).

${ }^{5}$ See, for example, Quantum Hall Effects, by T. Chakraborty and P. Pietiläinen (Springer, New York, 1995).

${ }^{6}$ S.-R. Eric Yang, A. H. MacDonald, and M. D. Johnson, Phys. Rev. Lett. 71, 3194 (1993).

${ }^{7}$ Kang-Hun Ahn, J. H. Oh, and K. J. Chang, Phys. Rev. B 52, 13757 (1995).

${ }^{8}$ C. W. J. Beenakker, Phys. Rev. Lett. 64, 216 (1990).

${ }^{9}$ D. B. Chklovskii, B. I. Shklovskii, and L. I. Glazman, Phys. Rev. B 46, 4026 (1992).

${ }^{10}$ For a review, see, for example, Field Theories of Condensed Matter Systems, by E. Fradkin (Addison-Wesley, Redwood City, 1991).

${ }^{11}$ E. Goldman and S. R. Renn, cond-mat/9606015 (unpblished); D. B. Chklovskii, Phys. Rev. B 51, 9895 (1995); for general theory see L. Brey, ibid. 50, 11861 (1994).

${ }^{12}$ P. Hohenberg and W. Kohn, Phys. Rev. 136, B864 (1964).

${ }^{13}$ M. Levy, Proc. Natl. Acad. Sci. USA 76, 6062 (1979).

${ }^{14}$ W. Kohn and L. J. Sham, Phys. Rev. 140, A1133 (1965).

${ }^{15}$ M. Levy, Phys. Rev. A 26, 1200 (1982); M. Levy and J. P. Perdew in Density Functional Methods in Physics edited by R. M. Dreizler and J. da Providencia (Plenum, New York, 1985).

${ }^{16}$ E. H. Lieb, Int. J. Quantum Chem. 24, 243 (1983).

${ }^{17}$ B. I. Halperin, Helv. Phys. Acta 56, 75 (1983).

${ }^{18}$ F. C. Zhang and T. Chakraborty, Phys. Rev. B 30, 7320 (1984).
${ }^{19}$ Density Functional Theory, by R. Dreizler and E. K. U. Gross (Springer Verlag, Berlin, 1990)

${ }^{20}$ F. D. M. Haldane, in The Quantum Hall Effect, edited by R. E. Prange and S. M. Girvin (Springer, New York, 1987).

${ }^{21}$ M. D. Johnson and A. H. MacDonald, Phys. Rev. Lett. 67, 2060 (1991).

${ }^{22}$ The original paper of Levy (Ref. 15) dealt with open-shell atoms with degenerate orbitals; for another ensemble extension of DFT to the so-called equiensembles of excited states, see E. K. U. Gross, L. N. Oliveira, and W. Kohn, Phys. Rev. A 37, 2805 (1988); ibid. 37, 2809 (1988).

${ }^{23}$ P. A. Maksym and T. Chakraborty, Phys. Rev. Lett. 65, 108 (1990).

${ }^{24}$ D. Levesque, J. J. Weis, and A. H. MacDonald, Phys. Rev. B 30, 1056 (1984).

${ }^{25}$ G. Fano and F. Ortolani, Phys. Rev. B 37, 8179 (1988).

${ }^{26}$ S. E. Barrett et al., Phys. Rev. Lett. 74, 5112 (1995); A. Schmeller, J. P. Eisenstein, L. N. Pfeiffer, and K. W. West, ibid. 75, 4290 (1995).

${ }^{27}$ S. L. Sondhi, A. Karlhede, S. A. Kivelson, and E. H. Rezayi, Phys. Rev. B 47, 16419 (1993).

${ }^{28}$ A. Karlhede, S. A. Kivelson, K. Lejnell, and S. I. Sondhi, Phys. Rev. Lett. 77, 2061 (1996).

${ }^{29}$ M. Franco and L. Brey, Phys. Rev. B (to be published).

${ }^{30}$ U. von Barth and L. Hedin J. Phys. C 5, 1629 (1972).

${ }^{31}$ R. Morf and B. I. Halperin, Phys. Rev. B 33, 221 (1986); N. d'Ambrumenil and R. Morf, ibid. 40, 6108 (1989).

${ }^{32}$ J. P. Perdew and A. Zunger, Phys. Rev. B 23, 5048 (1981).

${ }^{33}$ For a review, see R. O. Jones and O. Gunnarsson, Rev. Mod. Phys. 61, 689 (1989).

${ }^{34}$ A. Svane and O. Gunnarsson, Phys. Rev. Lett. 65, 1148 (1990); Z. Szotek, W. Temmerman, and H. Winter, Phys. Rev. B 47, 4029 (1993).

${ }^{35}$ G. L. Oliver and J. P. Perdew, Phys. Rev. A 20, 397 (1979).

${ }^{36}$ T. Chakraborty and F. C. Zhang, Phys. Rev. B 29, 7032 (1984); E. H. Rezay, ibid. 43, 5944 (1991). 